\title{
In Situ Observation of Quasimelting of Diamond and Reversible Graphite-Diamond Phase Transformations
}

\author{
J. Y. Huang* \\ Center for Integrated Nanotechnologies (CINT), Sandia National Laboratories, \\ Albuquerque, New Mexico 87185
}

Received April 26, 2007; Revised Manuscript Received June 20, 2007

\begin{abstract}
Because of technique difficulties in achieving the extreme high-pressure and high-temperature (HPHT) simultaneously, direct observation of the structures of carbon at extreme HPHT conditions has not been possible. Banhart and Ajayan discovered remarkably that carbon onions can act as nanoscopic pressure cells to generate high pressures. By heating carbon onions to $\sim 700{ }^{\circ} \mathrm{C}$ and under electron beam irradiation, the graphite-to-diamond transformation was observed in situ by transmission electron microscopy (TEM). However, the highest achievable temperature in a TEM heating holder is less than $1000^{\circ} \mathrm{C}$. Here we report that, by using carbon nanotubes as heaters and carbon onions as high-pressure cells, temperatures higher than $2000^{\circ} \mathrm{C}$ and pressures higher than $40 \mathrm{GPa}$ were achieved simultaneously in carbon onions. At such HPHT conditions and facilitated by electron beam irradiation, the diamond formed in the carbon onion cores frequently changed its shape, size, orientation, and internal structure and moved like a fluid, implying that it was in a quasimelting state. The fluctuation between the solid phase of diamond and the fluid/amorphous phase of diamond-like carbon, and the changes of the shape, size, and orientation of the solid diamond, were attributed to the dynamic crystallization of diamond crystal from the quasimolten state and the dynamic graphitediamond phase transformations. Our discovery offers unprecedented opportunities to studying the nanostructures of carbon at extreme conditions in situ and at an atomic scale.
\end{abstract}

The structure of carbon at extreme high pressures and high temperatures (HPHT), such as whether or not it is molten, ${ }^{1-6}$ is of interest to a wide variety research fields such as physics,${ }^{7}$ astronomy, ${ }^{8,9}$ geology, ${ }^{10}$ and materials sciences. ${ }^{11,12}$ The major hurdles in studying the structure of carbon at extreme pressure-temperature regimes lies in the experimental difficulties in achieving the extreme high pressures and high temperatures simultaneously. ${ }^{13-16}$ We found recently that a carbon nanotube can be Joule heated to temperatures higher than $2000{ }^{\circ} \mathrm{C}$ by passing a high current through it. ${ }^{17-21}$ It was also discovered previously that carbon onions $^{22,23}$ and carbon nanotubes can be used as high-pressure cells to generate high pressures. ${ }^{13,24-26}$ The question then arises: can we generate high pressures and high temperatures simultaneously by using carbon nanotubes as heaters and carbon onions as pressure cells?

Here we report that, by using carbon nanotubes as heaters and carbon onions as high-pressure cells, temperatures of $\sim 2000{ }^{\circ} \mathrm{C}$ and pressures higher than $40 \mathrm{GPa}$ were achieved in carbon onions, which led to the quasimelting of diamond and dynamic graphite-diamond transformations. Carbon

\footnotetext{
*E-mail: jhuang@sandia.gov.
}

onions usually coexist with nanotubes produced by arcdischarge, and these carbon onions are frequently attached to the nanotube surfaces by Van der Waals force. A twoterminal connection was made to individual nanotubes by using a Nanofactory transmission electron microscopyscanning tunneling microscopy (TEM-STM) platform. ${ }^{17-21}$ Once a connection was made, we then ran a high current through the nanotubes to heat the nanotubes and onions to high temperatures. From the melting and evaporation of highmelting-point ceramics, the graphitization of the nanotube walls, and the blackbody radiation spectroscopy, ${ }^{27}$ it was proved that a temperature of $\sim 2000{ }^{\circ} \mathrm{C}$ was reached in the nanotubes and carbon onions. ${ }^{17-21} \mathrm{We}$ then focused the e-beam to a current density of above $200 \mathrm{~A} / \mathrm{cm}^{2}$ on the carbon onions.

Under e-beam irradiation and at high temperatures, the hollow carbon onions (Figures 1a, 2a) continuously shrank until the inner hollows disappeared (Figures 1b, 2b). As the onion shrank, the inner shells were squeezed to an elongated (Figure 1b) or even a completely flattened shape (see, e.g., the innermost shell in Figure 2b). In the meantime, the overall shape of the carbon onions changed from polyhedral (Figures 1a, 2a) to elliptical (Figures 1b) or spherical (Figure 

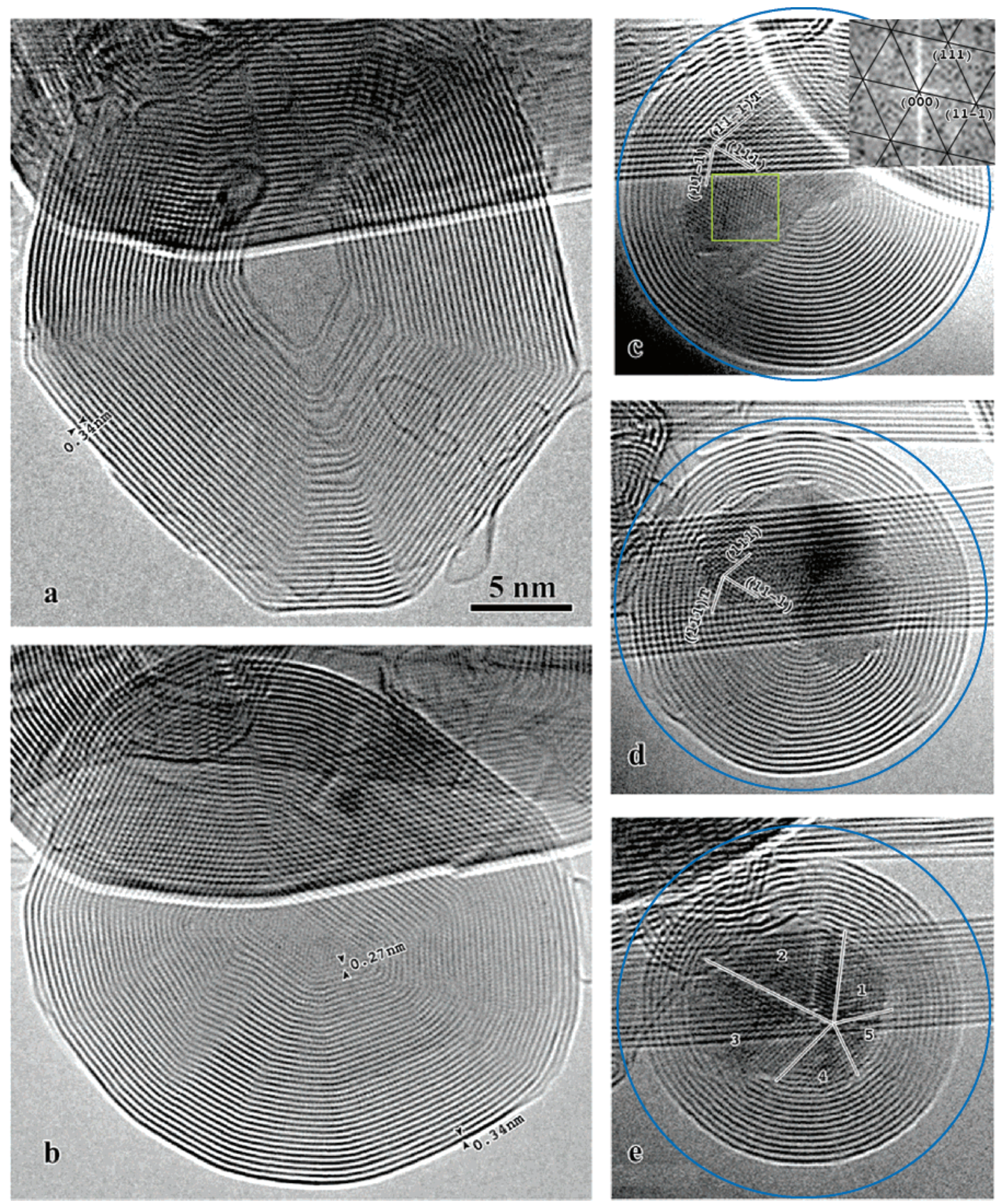

Figure 1. Sequential HRTEM images showing the shrinkage of a carbon onion and the formation of a diamond in the core at high temperatures and under e-beam irradiation. The onion was attached to a carbon nanotube (The nanotube was not shown in (a,b), but it can be seen in $(\mathrm{c}-\mathrm{e})$ ). The voltage applied to the nanotube was $2.5 \mathrm{~V}$. The dark contrast in the core of $(\mathrm{c}-\mathrm{e})$ corresponds to diamond. The time intervals in $(\mathrm{a}-\mathrm{e})$ are $0,2,10,15$, and $14 \mathrm{~min}$, respectively. The diameters of the carbon onions shown in $(\mathrm{c}-\mathrm{e})$ are 18.2, 17.4, and 16.4 nm, respectively. The blue circles having the same diameters are used as references to indicating the diameter reduction of the carbon onion. Inset in (c) is an FFT of the framed region in the same figure. A 5-fold twin is marked in (e). The diamond lattice planes are indicated.

2b). As the onions shrank, the number of shells was increased, e.g., from 33 (Figure 1a) or 23 (Figure 2a) to 38 (Figure 1b) or 31 (Figure $2 b$ ), respectively. The increase of the number of shells was duo to the growth of new shells from the inner shells but not from the outer surface. The radiation process induced knock-on displacements, collision cascades, and flux of carbon atoms toward the core, resulting in an increase of carbon atoms in the inner shells. At high temperatures, the atoms in the inner shells rearranged to form new shells. On the contrary, the outer shells were usually peeled off due to the sublimation of carbon atoms, a process similar to the peeling off of walls from a multiwalled carbon nanotube (MWCNT) ${ }^{18,28-30}$ With shrinking of the onion, the lattice spacing between the shells was reduced continuously from 0.34 to $0.27 \mathrm{~nm}$. The smallest spacing was measured to be $0.27 \mathrm{~nm}$ before a diamond was nucleated. According 

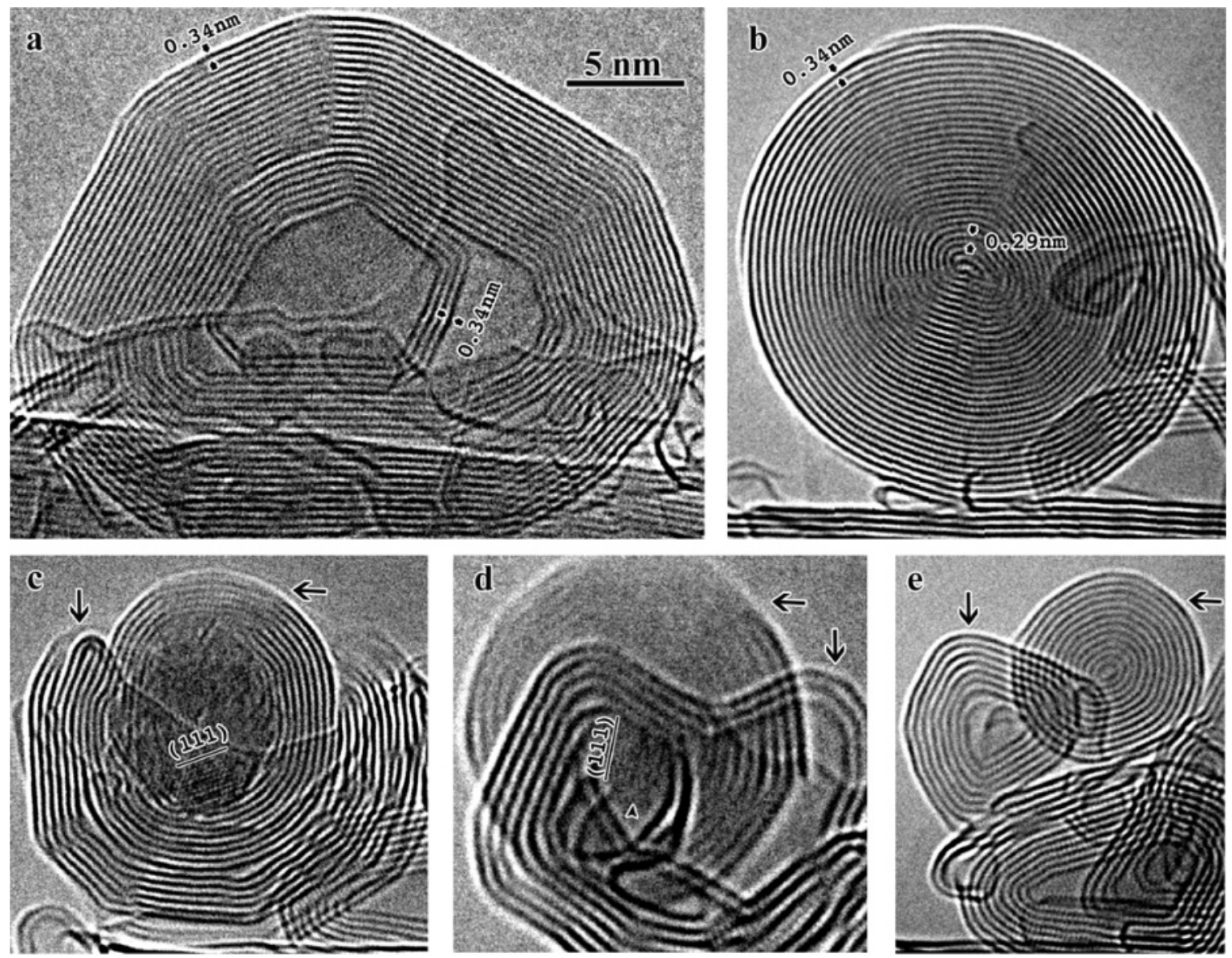

Figure 2. Sequential HRTEM images showing the shrinkage of a carbon onion and the formation of a diamond in the core at high temperatures and under e-beam irradiation. The onion was attached to the surface of a carbon nanotube. The voltage applied to the nanotube was $2.5 \mathrm{~V}$. The time intervals in (a-e) are $0,15,10,3$, and $2 \mathrm{~min}$, respectively. Note that the number of shells was increased from 23 (a) to 31 (b), and the innermost shell was flattened (b) as the onion shrank. As part of the onion shells were cut (c), the onion slipped out (d) and then the diamond core was converted back to carbon onion shells (e). The horizontal and vertical arrows point out the same onions, respectively. The arrowhead points out the diamond lattice fringes. The diamond lattice planes are indicated.

to the lattice spacing versus pressure plot, a pressure much higher than $40 \mathrm{GPa}$ is required to compress the $c$-axis spacing of graphite to $0.27 \mathrm{~nm}^{31}$

Once the diamond was nucleated, it frequently changed its shape, size, and orientation (Figures 1c-e, 3; Movies M1, M2, Supporting Information). The diamond usually exhibited a dark contrast and moved like a fluid; occasionally, lattice fringes or lattice images were captured in the video recording, which showed the formation of single crystals (Figures 2c, $3 \mathrm{c}, 3 \mathrm{e}$ ) or twinned crystals (Figures $1 \mathrm{c}, \mathrm{d}, 3 \mathrm{~b}$ ) or 5-foldtwinned crystals (Figure 1e) at different times. From the twodimensional lattice images (Figures $1 \mathrm{c}-\mathrm{e}, 3 \mathrm{~b}, 3 \mathrm{~d}$ ), the two sets of $\{111\}$ planes were clearly identified, and the lattice spacing was measured to be $0.2 \mathrm{~nm}$ and the angle between the two sets of $\{111\}$ planes was measured to be $70.5^{\circ}$, which match excellently with that of the diamond. Fast Fourier transformation (FFT) of the two-dimensional lattice images (e.g., framed area in Figure 1c) indicated that the electron diffraction pattern (inset in Figure 1c) is consistent with that of diamond. We have also conducted energy dispersive X-ray spectroscopy analysis, and carbon was the only element presented in the onion. These analyses confirmed conclusively that the condensed phase formed in the onion core was diamond.
As the number of the shells in the onion was decreased due to the sublimation of carbon atoms from the onion surfaces, the pressure in the onion was reduced but the temperatures remained at $\sim 2000{ }^{\circ} \mathrm{C}$. At high temperatures and ambient pressure, the diamond crystals were converted back to carbon onions (Figures 2d,e, 3e,f, Movie M2). We attempted to cut off part of the shells in the onion (Figure 2c) to see whether or not we could release the diamond core, but the onion slipped out (Figure 2d) before we could complete the cutting.

The quasimelting of diamond is well demonstrated in Figure 3 (Movie M2). The diamond crystal with a diameter of about $5 \mathrm{~nm}$ moved rapidly like a fluid, and occasionally lattice fringes or lattice images were detected. The diamond changed from a polycrystal (Figure 3a) to a multiple-twinned crystal (Figure 3b) to a single crystal (Figure 3c) to a twinned crystal again (Figure 3d) to a small single crystal (Figure $3 \mathrm{e}$ ) and finally to a carbon onion (Figure 3f). The fluidlike motion of the diamond crystal implied that the diamond was in a quasimelting state. The observation of the lattice fringes or images was due to the dynamic crystallization of diamond from the quasimolten state. We also observed reversible graphite and diamond transformation in the diamond-graphite interfaces (Figure 4). In this case, the 

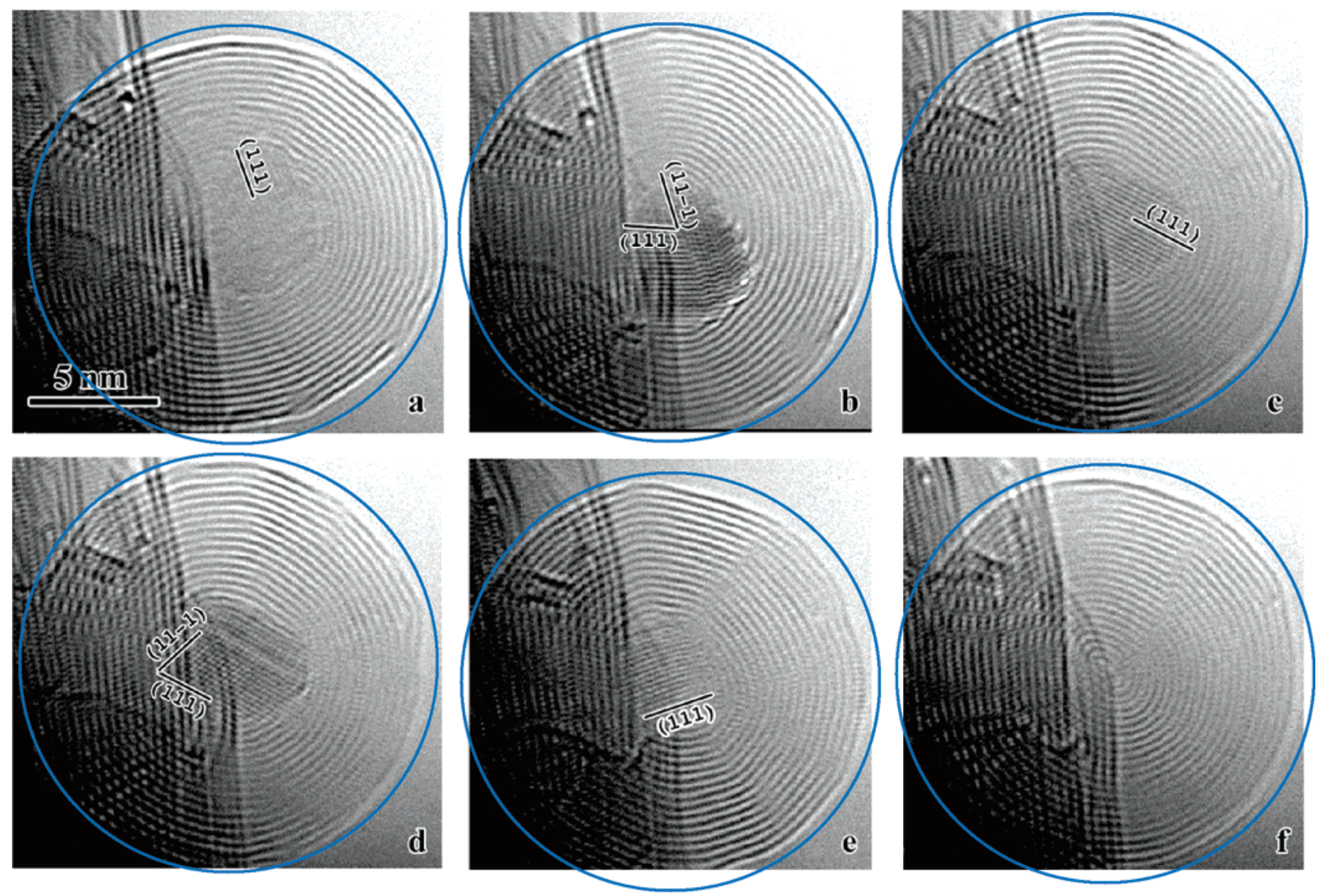

Figure 3. Sequential HRTEM images showing quasimelting of the diamond crystals. The shape, size, orientation, and internal structure of the diamonds changed continuously. In (f), the diamond was converted back to carbon onion shells. The time intervals in (a-f) are $0 \mathrm{~s}$, $2 \min 11 \mathrm{~s}, 32 \mathrm{~s}, 2 \mathrm{~s}, 26 \mathrm{~s}$, and $2 \min 23 \mathrm{~s}$, respectively. The blue circles are reference for the size and sphericity changes of the onions. The diamond lattice planes are marked.

graphite $\{0002\}$ planes were converted to the diamond $\{111\}$ planes and vice versa (Figure $4 d-f$ ).

Similar quasimelting behavior was observed in Au nanoparticles with free surfaces and with sizes less than $5 \mathrm{~nm} .{ }^{32-35}$ It was suggested that the state of such small nanoparticles should be called "quasisolid state," which is neither solid nor liquid. ${ }^{35}$ The quasimelting of $\mathrm{Au}$ nanoparticles was attributed to the high energetic e-beam irradiation ${ }^{35}$ or due to local heating induced by the e-beam..$^{33}$ The quasimelting of diamond cannot be explained by e-beam irradiation alone because, after cooling to room temperatures, the diamond was retained but its motion was stopped. The quasimelting behavior persisted even at a reduced e-beam dose, say at a regular image condition $\left(\sim 20 \mathrm{~A} / \mathrm{cm}^{2}\right)$, although the initial nucleation of diamonds required higher e-beam dose. We conclude that the source of the fluctuation between the solid diamond and the fluid diamond-like carbon was due to the diamond crystal crystallized from the quasimolten state and due to the dynamic graphite-diamond phase transformations induced primarily by the high temperatures in the nanotubes, although the quasimelting is facilitated by the e-beam irradiation.

We have the following convincing evidence showing that the temperature is over $2000{ }^{\circ} \mathrm{C}$ in the MWCNT at high bias voltages.

\section{Evaporation of $\mathrm{Al}_{2} \mathrm{O}_{3}$ Nanoparticles Attached to a} MWCNT. For temperature diagnostic purposes, we deposited $\mathrm{Al}_{2} \mathrm{O}_{3}$ nanoparticles on the surface of the nanotube walls (Figure 2 in ref 21). When increasing the bias to $1.2 \mathrm{~V}$, the catalyst Fe particle inside the MWCNT was melted and then flowed to the contact area; increasing the bias to $1.7 \mathrm{~V}$ caused melting of the $\mathrm{Al}_{2} \mathrm{O}_{3}$ nanoparticle, which was completely evaporated upon further increasing the bias voltage to $1.9 \mathrm{~V}$. The melting temperature of bulk Fe is $1540{ }^{\circ} \mathrm{C}$ and that of bulk $\mathrm{Al}_{2} \mathrm{O}_{3}$ is $\sim 2015^{\circ} \mathrm{C}$. The melting temperature of the $\mathrm{Al}_{2} \mathrm{O}_{3}$ nanoparticles $(\sim 20 \mathrm{~nm})$ should be similar to its bulk counterparts because size effect becomes significant only when the particle size is less than $10 \mathrm{~nm} \cdot{ }^{36}$ The sequential melting of $\mathrm{Fe}$ and $\mathrm{Al}_{2} \mathrm{O}_{3}$ (first $\mathrm{Fe}$ and then $\mathrm{Al}_{2} \mathrm{O}_{3}$ ) clearly indicated that the temperature was increased when the bias voltages were ramped up. ${ }^{21}$

2. Graphitization of Amorphous Carbon (a-C). It was found occasionally that the nanotube surface was coated with a-C (Figure S1a, Supporting Information). With increasing bias voltage to $2 \mathrm{~V}$, the initial a-C crystallized into turbostratic graphite (Figure S1b, Supporting Information), which reorganized into highly ordered nanotube walls upon further increasing of the bias voltage to $3 \mathrm{~V}$ (Figure S1c, Supporting Information). Temperature-controlled annealing of the amorphous carbon nanotube indicated that a temperature above 

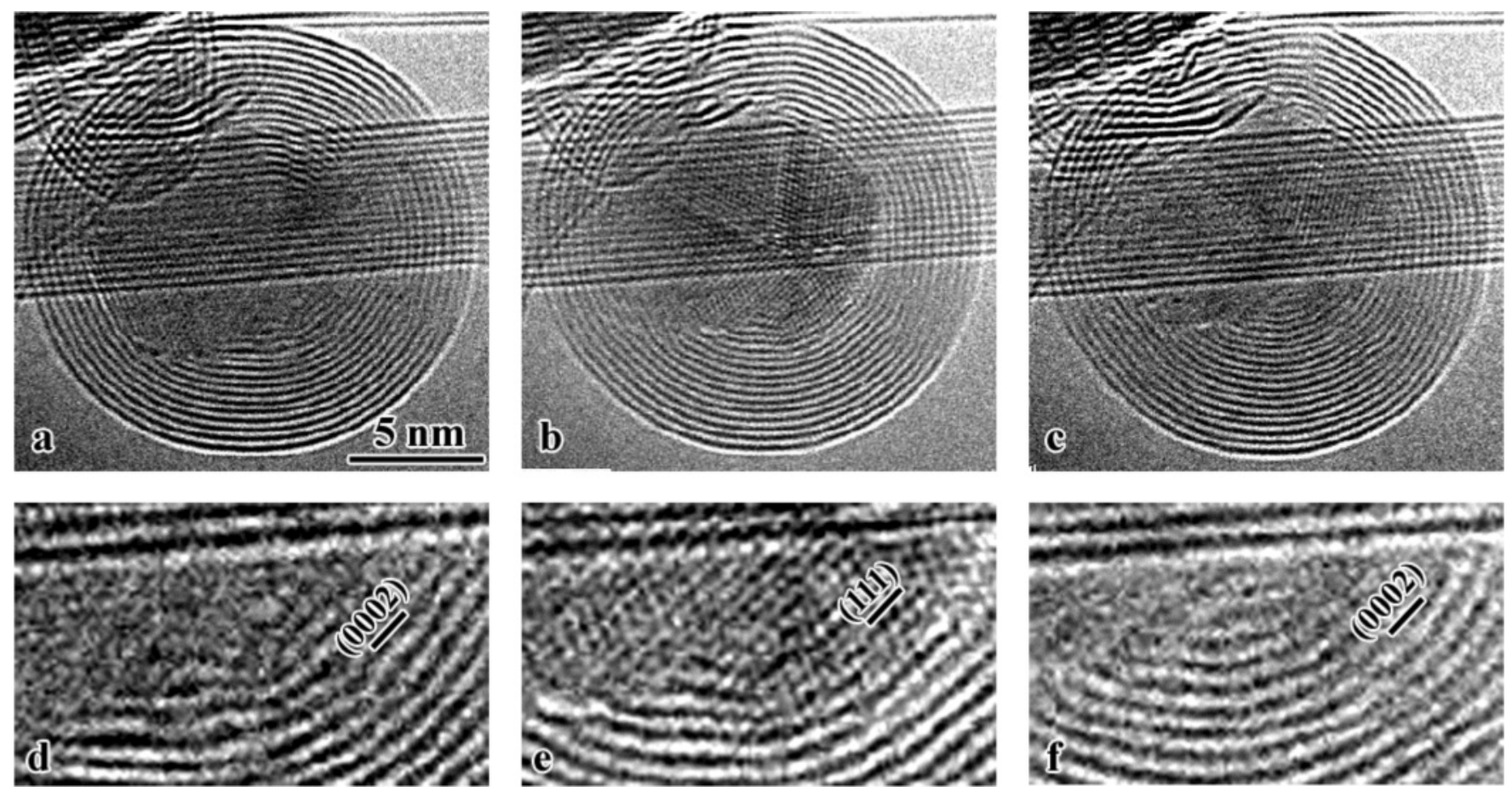

Figure 4. Reversible graphite and diamond transformation at the graphite-diamond interfaces. The graphitic (0002) planes (d) were converted to diamond (111) planes (e) and then converted back to graphitic (0002) (f) again. (d), (e), and (f) are local magnification of (a), (b), and (c), respectively.

$2000{ }^{\circ} \mathrm{C}$ was required to obtain highly ordered graphitic nanotube walls. ${ }^{37}$ Furthermore, perfect graphitization requires temperatures close to $3000{ }^{\circ} \mathrm{C} .{ }^{38}$ The crystallization of a-C to highly graphitized nanotube walls (Figure S1) at high bias voltages indicated that the temperature in the nanotube must be higher than $2000{ }^{\circ} \mathrm{C}$.

3. Black Body Radiation. ${ }^{27}$ When a high bias voltage is applied to a MWCNT, the MWCNT emits light through a black body radiation. Experiments showed that the radiation spectra were fitted excellently with a temperature of above $2000{ }^{\circ} \mathrm{C}$ at high bias voltages. ${ }^{27}$ Our experimental condition is very similar to that in ref 27 . Therefore it is concluded that the temperature in our MWCNT is higher than $2000{ }^{\circ} \mathrm{C}$ at high bias voltages.

The diamond melting line in the phase diagram of carbon is not clear. But according to an earlier phase diagram, $, 2,439$ diamond can be melted at $2000{ }^{\circ} \mathrm{C}$ and $\sim 50 \mathrm{GPa}$, and such a pressure and temperature is very close to that in our experiments. Furthermore, because of the high-energy ebeam radiation, the melting temperature of diamond could be reduced to much lower temperatures. Indeed, the onion to diamond transformation was observed at $\sim 700{ }^{\circ} \mathrm{C},{ }^{13,24}$ which is much less than that in a conventional HPHT experiments without e-beam radiation in which a temperature higher than $2000{ }^{\circ} \mathrm{C}$ and a pressure of above $10 \mathrm{GPa}$ are needed to convert graphite to diamond. ${ }^{2}$ In addition to the e-beam irradiation, the small grain size $(<10 \mathrm{~nm})$ might also contribute to the reduced melting temperature of diamond. But the main reason for the quasimelting of diamond is the high temperatures in the nanotubes because the quasimelting phenomenon disappeared when no voltage was applied to the nanotubes.
The extreme high temperatures required for melting diamond and graphite excludes any direct observation of the liquid phase thus far. The use of carbon nanotubes as heaters and carbon onions as high-pressure cells, combined with high-energy e-beam radiation, open new avenues to study the carbon phases at extreme pressure-temperature regimes in situ and at an atomic scale. Such studies may provide critical information regarding the carbon phases at extreme conditions, which is much needed in the fields such as condensed matter physics, ${ }^{7}$ geology, ${ }^{8,9}$ astronomy, ${ }^{10}$ and materials sciences. ${ }^{11,12}$ For example, we may be able to answer the fundamental question as to whether or not diamond or graphite melt under extreme conditions, ${ }^{1-6}$ which has important implications for studying the dynamics of the earth's mantle because the carbon in the lower mantle of the earth may be in a liquid form. ${ }^{8}$

Acknowledgment. I would like to thank Dr. S. Chen from Massachusetts Institute of Technology for making the STM probes, and Prof. M. Vaziri from University of MichiganFlint for providing the nanotube samples. J.Y.H. is on leave from Boston College. This work was performed, in part, at the Center for Integrated Nanotechnologies, a U.S. Department of Energy, Office of Basic Energy Sciences user facility. Supported by Laboratory Directed Research and Development (LDRD), Sandia is a multiprogram laboratory operated by Sandia Corporation, a Lockheed Martin Company, for the U.S. Department of Energy's National Nuclear Security Administration under contract DE-AC04-94AL85000.

Supporting Information Available: Crystallization of amorphous carbon to turbostratic graphite and to high- 
ordered nanotube walls when the bias voltages were ramped up in the nanotube. Movies of quasimelting of diamond at voltages applied to the nanotube of 2.5 and $2.55 \mathrm{~V}$ (QT and MPG). This material is available free of charge via the Internet at http://pubs.acs.org.

\section{References}

(1) Bundy, F. P. J. Chem. Phys. 1963, 38, 618.

(2) Bundy, F. P. Science 1962, 137, 1057.

(3) Whittaker, A. G. Science 1978, 200, 763.

(4) Gold, J. S.; Bassett, W. A.; Weathers, M. S.; Bird, J. M. Science 1984, 225, 921 .

(5) Heremans, J.; Olk, C. H.; Eesley, G. L.; Steinbeck, J.; Dresselhaus, G. Phys. Rev. Lett. 1988, 60, 452.

(6) Galli, G.; Martin, R. M.; Car, R.; Parrinello, M. Science 1990, 250, 1547.

(7) Bundy, F. P. Physica A 1989, 156,169.

(8) Dickey, J. S.; Bassett, W. A.; Bird, J. M.; Weathers, M. S. Geology 1983, 11, 219.

(9) Weathers, M. S.; Bassett, W. A. Phys. Chem. Mineral. 1987, 15, 105 .

(10) Ross, M. Nature 1981, 292, 435.

(11) Venkatesan, T.; Jacobson, D. C.; Gibson, J. M.; Elman, B. S.; Braunstein, G.; Dresselhaus, M. S.; Dresselhaus, G. Phys. Rev. Lett. 1984, 53, 360.

(12) Malvezzi, A. M.; Bloembergen, N.; Huang, C. Y. Phys. Rev. Lett. 1986, 57, 146.

(13) Banhart, F.; Ajayan, P. M. Nature 1996, 382, 433.

(14) Mcmillan, P. F. Nat. Mater. 2005, 4,715.

(15) Bridgman, P. W. The Physics of High Pressure; Bell: London, 1931.

(16) Hazen, R. M. The Diamond Makers; Cambridge University Press: New York, 1999.

(17) Huang, J. Y.; Chen, S.; Wang, Z. Q.; Kempa, K.; Wang, Y. M.; Jo, S. H.; Chen, G.; Dresselhaus, M. S.; Ren, Z. F. Nature 2006, 439, 281.
(18) Huang, J. Y.; Chen, S.; Jo, S. H.; Wang, Z.; Han, D. X.; Chen, G.; Dresselhaus, M. S.; Ren, Z. F. Phys. Rev. Lett. 2005, 94, 236802.

(19) Huang, J. Y.; Chen, S.; Ren, Z. F.; Wang, Z. Q.; Wang, D. Z.; Vaziri, M.; Suo, Z.; Chen, G.; Dresselhaus, M. S. Phys. Rev. Lett. 2006, 97 , 075501.

(20) Huang, J. Y.; Chen, S.; Ren, Z. F.; Chen, G.; Dresselhaus, M. S. Nano. Lett. 2006, 6, 1699.

(21) Chen, S.; Huang, J. Y.; Wang, Z.; Kempa, K.; Chen, G.; Ren, Z. F. Appl. Phys. Lett. 2005, 87, 263107.

(22) Kroto, H. W. Nature 1992, 359, 670.

(23) Ugarte, D. Nature 1992, 359, 707.

(24) Zaiser, M.; Banhart, F. Phys. Rev. Lett. 1997, 79, 3680.

(25) Sun, L.; Banhart, F.; Krasheninnikov, A. V.; Rodríguez-Manzo, J. A.; Terrones, M.; Ajayan, P. M. Science 2006, 312, 1199.

(26) Wang, Z. W.; Zhao, Y. S. Science 2006, 312, 1149.

(27) Cai, X. Y.; Akita, S.; Nakayama, Y. Thin Solid Films 2004, 464$465,364$.

(28) Yuzvinsky, T. D.; Mickelson, W.; Aloni, S.; Konsek, S. L.; Fennimore, A. M.; Begtrup, G. E.; Kis, A.; Regan, B. C.; Zettl, A. Appl. Phys. Lett. 2005, 87, 083103.

(29) Collins, P. G.; Arnold, M. S.; Avouris, Ph. Science 2001, 292, 706

(30) Bourlon, B.; Glattli, D. C.; Plaçais, B.; Berroir, J. M.; Miko, C.; Forró, L.; Bachtold, A. Phys. Rev. Lett. 2004, 92, 026804.

(31) Lynch, R. W.; Drickame, H. G. J. Chem. Phys. 1966, 44, 181.

(32) Bovin, J. O.; Wallenberg, R.; Smith, D. J. Nature 1985, 317, 47.

(33) Smith, D. J.; Petford-Long, A. K.; Wallenberg, L. R.; Bovin, J.-O. Science 1986, 233, 872.

(34) Ajayan, P. M.; Marks, L. D. Phys. Rev. Lett. 1989, 63, 279.

(35) Iijima, S.; Ichihashi, T. Phys. Rev. Lett. 1986, 56, 616.

(36) Buffat, Ph.; Borel, J.-P. Phys. Rev. A 1976, 13, 2287.

(37) Nishino, H.; Nishida, R.; Okimi, K.; Yokomichi, Y.; Matsui, T.; Mochida, I. Chem. Lett. 2004, 33, 162.

(38) Kelly, B.T. Physics of Graphite; Applied Science Publishers: London, 1981.

(39) Bundy, F. P. J. Geophys. Res. 1980, 85, 6930. NL0709975 